Meta

Journal des traducteurs

Translators' Journal

\title{
Le Projet Rideau Project : le théâtre " co-lingue ", le bilinguisme officiel et le va-et-vient de la traduction
}

\section{Nicole Nolette}

Volume 59, numéro 3, décembre 2014

Traduction et plurilinguisme officiel

Translation and Official Multilingualism

URI : https://id.erudit.org/iderudit/1028662ar

DOI : https://doi.org/10.7202/1028662ar

Aller au sommaire du numéro

Éditeur(s)

Les Presses de l’Université de Montréal

ISSN

0026-0452 (imprimé)

1492-1421 (numérique)

Découvrir la revue

Citer cet article

Nolette, N. (2014). Le Projet Rideau Project : le théâtre " co-lingue », le bilinguisme officiel et le va-et-vient de la traduction. Meta, 59(3), 654-672. https://doi.org/10.7202/1028662ar
Résumé de l'article

En 2009, le Théâtre la Catapulte, compagnie professionnelle francophone de création et de production théâtrale de la capitale nationale canadienne, montait un spectacle ambulatoire " co-lingue " où les spectateurs devaient circuler entre différents lieux du quartier central et historique du Marché By d'Ottawa. La composition de ce spectacle, le Projet Rideau Project, comptait six pièces de vingt minutes, dont trois étaient rédigées en français et trois en anglais, de six auteurs et metteurs en scène différents. Les négociations linguistiques au coeur du projet ont exigé l'apport de traducteurs de théâtre pour rééquilibrer la dynamique des langues dans le contexte de sa représentation : quatre pièces en anglais et deux pièces en français au festival de théâtre anglophone, et la proportion inverse au festival francophone. Selon sa propre appellation, « co-lingue », le Projet Rideau Project met en relief les enjeux dramaturgiques et traductologiques propres à la pratique et à la mise en scène de l'hétérolinguisme théâtral dans un contexte de bilinguisme officiel.
Ce document est protégé par la loi sur le droit d'auteur. L'utilisation des services d’Érudit (y compris la reproduction) est assujettie à sa politique d'utilisation que vous pouvez consulter en ligne.

https://apropos.erudit.org/fr/usagers/politique-dutilisation/ 


\title{
Le Projet Rideau Project: le théâtre «co-lingue», le bilinguisme officiel et le va-et-vient de la traduction
}

\author{
NICOLE NOLETTE \\ Université McGill, Montréal, Canada \\ nicole.nolette@mail.mcgill.ca
}

\section{RÉSUMÉ}

En 2009, le Théâtre la Catapulte, compagnie professionnelle francophone de création et de production théâtrale de la capitale nationale canadienne, montait un spectacle ambulatoire «co-lingue» où les spectateurs devaient circuler entre différents lieux du quartier central et historique du Marché By d'Ottawa. La composition de ce spectacle, le Projet Rideau Project, comptait six pièces de vingt minutes, dont trois étaient rédigées en français et trois en anglais, de six auteurs et metteurs en scène différents. Les négociations linguistiques au cœur du projet ont exigé l'apport de traducteurs de théâtre pour rééquilibrer la dynamique des langues dans le contexte de sa représentation: quatre pièces en anglais et deux pièces en français au festival de théâtre anglophone, et la proportion inverse au festival francophone. Selon sa propre appellation, «co-lingue», le Projet Rideau Project met en relief les enjeux dramaturgiques et traductologiques propres à la pratique et à la mise en scène de l'hétérolinguisme théâtral dans un contexte de bilinguisme officiel.

\begin{abstract}
In 2009, francophone theatre company Théâtre la Catapulte, whose mandate is to create and produce theatre in Canada's national capital, staged a site-specific "colingual" performance in which spectators walked between different stage spaces in Ottawa's central and historical By Market. The components of this performance, le Projet Rideau Project, were six twenty-minute plays, three of which were in French, three in English, by six different authors and directors. The linguistic negotiations at the heart of the project required theatre translators to balance the language dynamics in the context of the performance: four plays in English and two in French at an English-language theatre festival, and the opposite proportion for a French-language festival. Self-declared by its instigators as "colingual," le Projet Rideau Project showcases the stakes around writing, staging, and translating heterolingual theatre in a context of official bilingualism.
\end{abstract}

\section{MOTS CLÉS/KEYWORDS}

hétérolinguisme, traduction, théâtre, Canada, bilinguisme officiel heterolingualism, translation, theatre, Canada, official bilingualism

\section{Ottawa : tensions, rencontres et bilinguisme officiel}

À titre de capitale du Canada, une nation reconnue pour son bilinguisme officiel, ainsi que de centre névralgique de la pratique de la traduction, la ville d'Ottawa semble porteuse de diverses avenues pour le bilinguisme et la traduction. En effet, c'est à partir d'Ottawa qu'ont été instaurés les articles 4, 5 et 14 de la Loi sur les langues officielles, adoptée en 1969. Ces trois articles visent à assurer le droit à l'usage du 
français et de l'anglais dans les affaires du Parlement et des tribunaux fédéraux, l'adoption des lois dans les deux langues officielles et le droit à l'interprétation simultanée. La plupart des institutions fédérales, auxquelles il incombe de communiquer avec les citoyens dans la langue officielle de leur choix (Article 21), sont aussi situées à Ottawa ou sur la rive opposée, du côté du Québec, à Gatineau. Incidemment, la pratique institutionnelle de la traduction, qui assure l'accès des citoyens aux services gouvernementaux dans la langue officielle de leur choix, a souvent agi de manière à minimiser le côtoiement quotidien des langues. Dans ce régime du bilinguisme officiel, la traduction assure le maintien de l'unilinguisme des citoyens. Ainsi, dans la capitale nationale, la production artistique et théâtrale s'est le plus souvent tenue aux confins d'une langue officielle ou de l'autre. La ville d'Ottawa compte donc au moins deux communautés théâtrales définies selon leur affiliation linguistique ${ }^{1}$. Les comédiens, metteurs en scène, dramaturges, régisseurs et autres professionnels de théâtre d'expression anglaise alimentent le milieu anglophone, tandis que ceux dont la langue est le français gravitent habituellement autour du milieu francophone.

Le milieu théâtral francophone ottavien s'est d'ailleurs reconstitué dans la foulée des débats autour de la Loi sur les langues officielles et de sa mise en pratique. Répondant à la redéfinition de la nation canadienne-française d'après les frontières géographiques du Québec, les artistes francophones d'Ottawa comme ceux de l'Ontario français ont créé, au cours des années 1970-1980, un théâtre qui pouvait répondre aux nouvelles lignes d'appartenance identitaires. Ces lignes d'appartenance se dessineraient selon des délimitations linguistiques et géographiques - celles de l'Ontario et de la langue française -, ces délimitations étant constituées par opposition aux Autres précis. La critique littéraire Lucie Hotte (2000: 163-178) le fait valoir au sujet des Franco-Ontariens: «c'est dans le rapport à l'Autre que les traits identitaires deviennent actifs. Le francophone, par exemple, ne peut être francophone qu'en relation avec d'autres groupes linguistiques ", en l'occurrence, la majorité anglo-canadienne. De même, les Franco-Ontariens se définissent par rapport à la frontière géographique avec le Québec, frontière dont la présence tangible à Ottawa, «assez artificielle sur le plan régional, allait aussi devenir pour la nation québécoise et la communauté franco-ontarienne nouvellement conçues une frontière culturelle et identitaire de plus en plus étanche» (Beddows 2001: 50). Dans un premier temps, le théâtre francophone d'Ottawa se mobilise grâce à cette double altérité constitutive; de son côté, le théâtre anglophone de la ville évolue en périphérie des métropoles torontoise et new-yorkaise. La "superposition» des deux milieux théâtraux sur la cartographie de la ville d'Ottawa aurait cependant maintenant, selon la critique de théâtre Stéphanie Nutting (2012: 13), une incidence majeure sur «la carrière d'une poignée de praticiens de théâtre bilingues qui [y] œuvrent». Parmi ceux-ci, elle identifie Joël Beddows comme «un des rares metteurs en scène biculturels qui savent naviguer et exploiter avec aisance les labyrinthes de l'espace double» (Nutting 2012: 13).

Directeur artistique du Théâtre la Catapulte, compagnie professionnelle de création, de production et de diffusion d'Ottawa, de 1998 à 2010, professeur au Département de théâtre de l'Université d'Ottawa, Joël Beddows est en effet l'un des instigateurs de la traversée toujours inhabituelle des communautés théâtrales de langues officielles de la capitale nationale. Avant de quitter son poste de directeur artistique de la Catapulte, et avec la collaboration de Mary Vingoe du Magnetic North Theatre 
Festival et de Paul Lefebvre de la biennale Zones Théâtrales, il organise en 2009 le spectacle d'envergure Projet Rideau Project ${ }^{2}$. Ce spectacle ambulatoire dit «colingue» dans ses matériaux publicitaires, le Projet Rideau Project - une double allusion au canal Rideau qui traverse le centre-ville d'Ottawa et au rideau de théâtre -, est habité des traversées géographiques, linguistiques et théâtrales de la ville. Selon l'objectif déclaré, les créateurs souhaitent «explorer, sur le plan thématique, les "histoires perdues" de la capitale nationale ${ }^{3} »$. Par-delà la récupération historique, un désir de cohabitation linguistique et théâtrale de la ville semble sous-tendre le projet pour lequel Beddows, à la tête de la Catapulte, a rassemblé plus de quatre-vingts artistes de la région d'Ottawa (dont Gatineau). Dans son mot du directeur artistique, Beddows souligne à quel point "[i]l s'agit d'un moment de contact et d'échange artistique» entre deux «"façons" dont les deux groupes culturels et les artistes choisissent aussi de raconter ${ }^{4} »$. Auteurs, metteurs en scène, régisseurs, traducteurs, conseillers dramaturgiques, comédiens, responsables des costumes et de l'environnement sonore, de langue française ou anglaise, tous sont conviés à participer à la production d'un spectacle où les spectateurs doivent circuler entre différents lieux du quartier central et historique du Marché By.

Le spectacle compte six pièces d'une vingtaine de minutes, dont trois rédigées en français (Cercles polaires de Michel Ouellette; Bison mystique de Luc Moquin; Rebut de Sarah Migneron) et trois en anglais (Tourist Things de Patrick Gauthier; Peace, Land and Bread de John Ng; The Rhyme of Nicholas Street Goal de Pierre Brault). Chaque pièce en français est montée par un metteur en scène de la communauté anglophone et vice versa. Pour assurer la présentation d'un spectacle d'une telle envergure, et réparti dans autant de lieux, la Catapulte s'allie au Magnetic North Theatre Festival, le festival de nouvelle dramaturgie canadienne en anglais, à Zones Théâtrales, un rassemblement de théâtres professionnels des communautés francophones canadiennes et des régions du Québec, et à la Commission de la capitale nationale, une société d'État dont le mandat, dans la Capitale-Nationale, est d'«organiser, de parrainer et de promouvoir des activités et des manifestations publiques qui reflètent le patrimoine [du] pays, et ce, dans le respect des langues officielles ${ }^{5} »$. Ces partenariats agissent à la fois sur les représentations du spectacle (sept représentations au Magnetic North Theatre Festival en juin 2009, trois à Zones Théâtrales en septembre 2009) et sur la distribution des langues dans les six pièces. Lors du Magnetic North Theatre Festival, quatre des textes sont joués en anglais, deux en français; pendant Zones Théâtrales, la proportion est inversée pour que deux textes soient présentés en anglais et quatre en français. Dans ces deux festivals, ce sont les pièces d'ouverture et de clôture du spectacle, Tourist Things et Rebut, qui sont traduites: Pour les touristes et Trash. Les négociations linguistiques au cœur du projet exigent l'apport de traducteurs de théâtre pour rééquilibrer la dynamique des langues dans le contexte de sa représentation: Paul Lefebvre pour Tourist Things et Paula Danckert pour Rebut. Mais puisque le Projet Rideau Project demeure, dans toutes ses représentations, un projet «co-lingue», il exige aussi des dispositifs «ambulatoires» de traduction et de non-traduction pour rendre possible le trajet de spectateurs dont les compétences linguistiques varient.

Je propose ici un parcours des enjeux traductologiques propres à l'hétérolinguisme théâtral dans le contexte du bilinguisme officiel canadien, et plus particulièrement dans la capitale nationale. Cet itinéraire suivra les routes tracées par 
l'expérience de création et de traduction du Projet Rideau Project de même que ses accidents de parcours. Aux analyses dramaturgiques et traductologiques du projet s'ajoutera une analyse de la juxtaposition délibérée des textes d'auteurs dramatiques d'une culture théâtrale de langue officielle et des mises en scène de l'autre culture. Au terme de ces déambulations, il y aura lieu de remettre en question les distinctions établies entre les concepts du bilinguisme officiel canadien, de l'hétérolinguisme - néologisme créé par Rainier Grutman (1997) pour différencier le plurilinguisme littéraire de ses manifestations sociales et politiques - et du co-linguisme sous lequel le Projet Rideau Project prend l'affiche.

\section{Le Projet Rideau Project: un parcours déambulatoire}

Pour assister au spectacle du Projet Rideau Project, les spectateurs sont invités à un premier point de rencontre devant le Musée des beaux-arts, sous la gigantesque sculpture araignée Maman de Louise Bourgeois. Ils prennent place sur une trentaine de chaises pliantes qui entourent les huit pattes de l'araignée de 10 mètres de hauteur et de largeur. Une jeune femme vêtue d'un manteau brun s'installe sur un banc en demi-lune sous l'araignée, son appareil photo fixé vers le haut dans un effort de cadrage pour Tourist Things de Patrick Gauthier. Un homme arrive et se présente à la jeune femme en la confondant avec une autre. Elle lui demande de la prendre en photo, lui annonce qu'elle est fiancée. Ils se rendent compte tous les deux qu'on les a oubliés, que la personne qu'ils attendent ne viendra pas. Ils se rapprochent, prennent d'autres photos et s'embrassent avant que le fiancé n'arrive et que le secret de la femme ne soit dévoilé: elle joue le rôle d'une autre, espérant vivre des instants d'un bonheur éphémère comme celui d'une photographie prise par un bel étranger. La femme repart avec son faux fiancé, l'homme avec la pellicule des photos issues de cette rencontre, et les spectateurs s'en vont avec le guide pour voir la prochaine pièce à la cour Jeanne-d'Arc du Marché By, entre les rues Clarence et York.

Dans cette cour publique, deux sculptures, l'une d'un ours dansant, l'autre d'une jeune fille tenant un cerceau au-dessus de sa tête, ont inspiré la pièce de Michel Ouellette, Cercles polaires. Les deux personnages, une jeune femme vêtue à l'ancienne qui dessine des cercles dans son carnet et un homme dont les vêtements trop grands le font trébucher, sont eux aussi en attente. Elle attend un homme depuis une semaine pour monter dans le Grand Nord voir le dernier ours polaire; il attend son frère. Après des tentatives de rapprochement et des démonstrations de cerceau, les deux se rendent à l'évidence qu'ils pourraient rester très longtemps figés dans cette vaine attente. Incapables de faire autre chose que d'en témoigner, les spectateurs se déplacent vers le Centre des congrès d'Ottawa pour assister à la pièce de John Ng, Peace, Land and Bread. Nommée d'après le slogan léniniste, elle reprend l'anecdote historique de l'affaire Gouzenko: Igor Gouzenko, un fonctionnaire de l'ambassade soviétique, frappe aux portes de l'édifice du ministère de la Justice en septembre 1945. Il souhaite faire défection et porte avec lui des documents secrets attestant un réseau d'espionnage soviétique en Amérique du Nord. Le commissionnaire qui est de service ce soir-là refuse de le croire et ne leur offre, à lui et à son épouse enceinte, qu'un bout de pain acheté dans une boulangerie ukrainienne. La pièce se solde par cet échec de la communication qui laisse pressentir l'amorce de la guerre froide. 
L'absurdité de la communication et de l'attente reprend son cours sous le pont Plaza dans Bison mystique de Luc Moquin. Comme le personnage féminin de Cercles polaires, les deux cow-boys de Bison mystique attendent depuis une semaine. Leur attente est un peu plus active, cependant, puisqu'ils cherchent la piste du bison pour se remettre sur les traces de cet animal mystique. Ils tombent alors sur une aubergiste éméchée, nostalgique de son saloon Chez Guert, et un Big Bill menaçant à qui ils sont obligés de donner une de leurs bottes. Le battement de la piste de bison se laisse toujours désirer, et les cow-boys peinent à aller plus loin ainsi mal bottés. Tel n'est pas le cas pour les spectateurs: un guide les mène vers une cellule dans le sous-sol de la Cour des arts, lieu historique de la dernière pendaison d'Ottawa. The Rhyme of Nicholas Street Goal met en scène les vingt dernières minutes de la vie d'un pendu, Eugène Larmont. Pendant le décompte final, le prisonnier demande une dernière tasse de thé, déchire les pages d'une bible, raconte ses vingt-quatre années dans un foyer violent et ses rencontres répétées avec les forces de l'ordre. Il refuse de faire acte de foi, malgré les supplications d'une sœur de la Charité. Par son poème dramatique, il confesse ses crimes mais il ne demande pardon à personne.

Les spectateurs n'auront pas à se déplacer plus loin que l'allée entre la Cour des arts et l'ancienne prison pour que la dame sans abri de Rebut les saisisse au passage - ou plutôt, elle intercepte des passants invisibles aux spectateurs, mais auxquels ceux-ci pourraient facilement s'identifier. Elle tend son gobelet à ces passants, leur récite des blagues et des histoires afin de veiller à la survie d'une poupée qu'elle prend pour son enfant. Puis elle raconte son histoire, la transformant en conte de fées pour endormir la poupée et dévoilant les conditions du décès de sa fille Mélodie. Accusée par le personnage d'une passante généreuse de jouer le jeu de la culpabilité, elle ferme la clôture de l'allée et la transforme en huis clos. Afin de s'assurer du juste châtiment de ses méfaits, la dame enfonce un couteau dans le flanc de la femme charitable. Dans l'ensemble des pièces du spectacle, le Projet Rideau Project trouve ainsi un dénouement tragique, le désir de rencontre des étrangers de Tourist Things se soldant au terme des déambulations par la violence d'un contact pourtant d'abord généreux dans Rebut, après être passé par l'attente de Cercles polaires, de Bison mystique et de The Rhyme of Nicholas Street Goal, puis par le refus d'écouter de Peace, Land and Bread.

\section{Le Projet Rideau Project: des langues qui résonnent ?6}

Le Projet Rideau Project propose déjà, dans son intégralité flexible, un cas d'hétérolinguisme théâtral intéressant. Des six pièces qui composent le projet, trois pourraient être catégorisées comme étant monolingues: Tourist Things, Cercles polaires et Rebut. Ce sont par ailleurs deux de ces pièces, Tourist Things et Rebut, qui étaient traduites à l'occasion des festivals. Tourist Things présente la rencontre entre les deux inconnus dans une langue anglaise oralisée qui devient rapidement informal à la suite de leur rapprochement:

(1) Woman: Can I take one last photo? Of us? It would be nice to have a photo of the two of us.

Man: I don't think so. I don't think it's a good idea. 
À certains moments, évoquant Paris et en mode séduction, l'homme dit ses répliques avec un accent français: «Think. Think about it. If you live in France - in Paris - you don't ask a stranger to take your photo in front of the Eiffel Tower» (Gauthier 2009a: 5; voir note 7). La mendiante de Rebut parle un français qui déjoue le pacte de la représentation linguistique établi par Tourist Things, oscillant entre les niveaux de langue courant et soutenu, et acquérant des notes de littérarité lorsque le personnage se met à raconter son conte de fées au passé simple. Elle narre l'histoire à sa poupée-enfant:

(2) Il était une fois, il n'y a pas si longtemps et tout près d'ici, une jeune fille qui naquit un violon à l'épaule et un archet à la main. Tout le jour, toute la nuit, cette jeune fille ne rêvait qu'à une chose: envoûter le monde avec sa musique.

(Migneron 2009a: $4^{8}$ )

Dans Cercles polaires, Michel Ouellette s'éloigne lui aussi du réalisme linguistique pour aller vers une écriture lyrique. La langue de ses deux personnages se plie aux formes dansantes et circulaires des statues de la cour Jeanne-d'Arc. Saccadée, répétée et rythmée, elle tourne en rond et s'arrête aussi abruptement qu'un jeu de cerceau. Le discours de la jeune femme sur la perte joue sur l'allitération plutôt que de sombrer dans la psychologie du personnage:

(3) Non. Non. Non... Il m'a fait de si belles promesses. Il parlait si bien. Avec une telle assurance dans la voix. Il parlait, j'écoutais. Je me sentais bien dans sa grande bouche voluptueuse. J'étais un caramel sur sa langue. Un petit caramel sucré. Mais là je suis complètement dissoute. Je suis dissuadée. Je suis déçue. Trop déçue pour continuer.

(Ouellette 2009: 119)

Rien dans la langue poétique des pièces de Migneron et de Ouellette ne laisse deviner le code linguistique local ou l'appartenance à la ville d'Ottawa. De fait, pour Ouellette, et la critique Ariane Brun del Re (2012: 130) l'a fait valoir au sujet de sa pièce King Edward (1999), «la ville d'Ottawa ne [...] paraît pas suffisamment accueillante envers sa minorité francophone». Dans Cercles polaires, Ouellette abandonne la partie: l'attente beckettienne de ses personnages leur nie tout attachement à la ville dans laquelle ils se situent, si ce n'est que pour les statues de la cour Jeanne-d'Arc. Comme dans les deux autres pièces écrites en français, les personnages sont des figures marginalisées, des laissés-pour-compte. Ces individus, dont les expériences ottaviennes sont pourtant des «histoires perdues», répondant à la commande du Projet Rideau Project, content l'histoire d'une minorisation linguistique urbaine sans pour autant que cette dernière soit rattachée à une ville particulière. Le particularisme de telles histoires est axé sur «les thèmes franco-ontariens traditionnels, telles l'assimilation, la marginalisation et l'aliénation» (Hotte 2002: 44); cependant, il ne met pas en scène les Franco-Ontariens, leur espace ni leur parler. De fait, il trace une ligne de fuite s'opposant diamétralement au courant individualiste repéré par Hotte (2002) dans la littérature franco-ontarienne contemporaine, par lequel les thèmes fétiches laissent leur place à l'intégration harmonieuse de repères franco-ontariens dans le texte littéraire.

En revanche, dans Bison mystique, on retrouve un hétérolinguisme marqué tant par l'insertion d'idiomes étrangers que par celle des variétés sociales, régionales ou 
historiques de la langue principale (Grutman 1997). Figures ridicules, clichés du tourisme de l'Ouest canadien comme des westerns-spaghettis, les cow-boys de Luc Moquin parlent un français canadien parsemé d'emprunts à l'anglais pour certaines références incontournables au Far-West:

(4) Comment? Si c'est encore loin? Je cré ben, mes chers, que c'est encore loin, mais c'est bien difficile à dire, vu qu'on sait plus pantoute c'est par ousque-cé qu'on s'en va. Voyez-vous, l'autre imbécile qu'il y a là-bas, ben ça fait une semaine qu'y nous dit qu'y sait où aller, mais y finit toujours par nous ramener à la même place. On avait une bonne trail, pis tout ce qu'on avait à faire, c'était la suivre.

(Moquin 2009: $2^{10}$ )

À leur variété de français s'ajoute celle de l'aubergiste. Non seulement cette femme mûre est-elle complètement ivre, mais son dialecte accentue (aux deux sens du terme) l'effet comique de sa présence. Son dialecte résonne avec celui de la plus grande figure du théâtre acadien, la Sagouine (Maillet1971'11). À cette dernière, l'aubergiste emprunte des caractéristiques linguistiques comme la conjugaison de la première personne du pluriel pour la première personne du singulier («j'étions»). Réveillée d'un sommeil éthylique par les cow-boys et se présentant comme hôtesse de saloon, l'aubergiste raconte: «Tous les hommes y venant prendre leur petit coup l'après-midi, pour jaser avec Guert. Et moi, vous comprenez, je leur faisions des belles manières, affaire de faire rouler la business. Y me contaient leur histoire, pis moi je les écoutions, j'étions toujours ben bonne dans l'écoutage» (Moquin 2009: 8; voir note 10$)$.

L'aubergiste éméchée, parodie de la Sagouine bien candide d'Antonine Maillet, est traversée par une autre parodie de cette même figure. Il s'agit de la Sagouine du comédien québécois Marc Labrèche, créée pour une série de sketchs de l'émission humoristique 3600 secondes d'extase, diffusée sur les ondes de Radio-Canada de 2008 à 2011. Dans cette série de sketchs, la «chanson des Prairies» - diffusée après la production du Projet Rideau Project mais répondant au même humour -, Labrèche revisite le rôle de la Sagouine. En portant les «hardes» distinctives de cette dernière, il chante des paroles qui évoquent les Prairies des cow-boys de Moquin:

(5) J'avions connu des cochonnes

Des filles de petite vertu

Mais, les pires j'avions connu

V’nions du Québec et des Prairies [...]

À toutes les guidounes de Saskatoon [...]

Faisions le Kama Sutra à Moose Jaw

Mais usions pas les draps à Ottawa ${ }^{12}$.

Et comme les «filles de petite vertu» qui «usions pas les draps à Ottawa», ni l'aubergiste ni les cow-boys de Moquin n'investissent la capitale nationale. Pourtant, l'auteur convoque deux stéréotypes anachroniques des extrémités est et ouest du Canada. Qui plus est, ces deux figures pourraient resolidariser la francophonie canadienne en investissant la ville d'Ottawa, à la fois comme espace scénique et comme capitale culturelle en puissance. On pourrait même voir dans le Bison mystique une mise en abyme ludique et critique du festival franco-canadien dans lequel on présentait le Projet Rideau Project, Zones Théâtrales. Or, malgré l'utopisme polisson de ces 
allusions, il reste que le lieu de rencontre entre l'est et l'ouest du Canada, qui ne peut être que la capitale nationale, est aussi un lieu où les personnages n'ont rien à faire, rien à se dire, où ils sont dans l'inertie la plus totale.

\section{Le Projet Rideau Project: des langues qui détonnent?}

De fait, au creux de cette inertie, une seule figure peut faire sursauter les cow-boys et l'aubergiste: celle du gros méchant Big Bill, décrit comme un «[p]rêteur sur gage, brutal et lâche (et anglophone)»(Moquin 2009: 1; voir note 10). Big Bill s'inscrit ainsi dans une longue lignée littéraire au Québec comme au Canada français, celle des étrangers anglophones dont il faut se méfier. Au sujet des romans du mouvement québécois partipriste des années 1960, Simon Harel (1989: 103) explique que l'étranger anglophone y «sert avant tout de repoussoir. Il incarne une menace, le danger d'une domination.» Cette menace est habituellement liée, comme dans Bison mystique, aux inégalités économiques entre les groupes linguistiques: les cow-boys doivent trente dollars à Big Bill.

(6) Bill: Or what? You're gonna kick me with your sissy boots, is that it?

CB1 : Écoute Bill, demain on va l'avoir ton cash, promis, mais...

Bill: No you listen. I've had it with you stupid fucks who think the world is free. You think I'm just giving my money away? Like I'm some kind of charity? Here's a news check for you, cowgirl: I don't care about you, what I want is my money. [...]

CB2: Bon, ça va faire là. On t’avait donné nos ceintures à garder en garantie au cas où on pourrait pas te payer, pis après ça on t'a donné une belle montre. On l'a remboursé ben en masse ton trente piasses, Bill, fait que laisse-nous tranquilles.

(Moquin 2009: 16-17; voir note 10)

Grâce à la convention théâtrale adoptée, celle d'un bilinguisme par lequel les interlocuteurs comprennent davantage l'autre langue qu'ils ne la parlent, les personnages arrivent à communiquer entre eux pour régler la dette. Or, si la langue anglaise et la figure américaine de Big Bill dans cette pièce semblent affreusement menaçantes, il est presque impossible de les prendre au sérieux. Le portrait de l'étranger anglophone des romans partipristes est poussé à l'extrême du cliché et les enjeux de la domination qu'il soulève sont tournés en dérision et mis à nu dans leur grossièreté. Big Bill part ainsi avec une botte rouge et une botte noire dans les mains et les cowboys se partagent une carotte.

Mais alors même que Bison mystique en fait une caricature, la valeur des enjeux de la domination linguistique n'est que réaffirmée par la prochaine pièce, The Rhyme of Nicholas Street Goal. Pierre Brault fait intervenir ses personnages francophones dans leur langue, mais seulement pour donner un effet de réel. Le vocabulaire français est réservé à des référents facilement identifiables pour un public qui ne comprend pas cette langue. Des interjections telles les «Oui, monsieur» de la sœur de la Charité et les «patron» du prisonnier confirment la domination anglophone (Brault 2009: 12, 10 13). En allant au-delà de ces confins, cependant, les personnages francophones attirent la colère de l'assistant de l'exécution (No. 2), assureur à ses heures: 
(7) Eugène: Attendez une minute, s'il vous plaît, ma Sœur!

Hélène: Oui, Monsieur?

Eugène: Le curé, où est-il?

No. 2: Hold on now. No French! That's the rules.

Eugène: I'm asking her where the priest is.

Goale: Let him speak his mother tongue.

No. 2: English only, those are the rules. What if he says something secret to her? Eugène: Il ne faut jamais acheter de l'assurance d'un petit homme.

No. 2: What did you say to her? What did he say to you?

Goaler: You leave her alone. Understand?

(Brault 2009: 12-13; voir note 13)

Dans cette scène, le No. 2 se fait exécuteur des règles linguistiques. Et lorsque le geôlier plaide en faveur du prisonnier, l'assistant lui rappelle que dans les zones opaques de l'autre langue il existe aussi des zones propices au secret. C'est ce rappel, de fait, qui provoque l'insulte du prisonnier à l'égard du No. 2, insulte qu'il se fait un plaisir de faire dans l'opacité du français. Le geôlier ne le comprend pas, la religieuse ne le dénonce pas; les deux le protègent des règles systémiques comme de leur exécution par le No. 2. En évoquant ainsi sa peur de la langue étrangère comme code secret entre le prisonnier et la religieuse, le No. 2 impose une censure linguistique interne à la pièce The Rhyme of Nicholas Street Goal.

Dans un dialogue subséquent entre la religieuse et le prisonnier, en l'absence des gardes, c'est en anglais que les personnages discutent. Le No. 2 devient ainsi, effectivement, ce à quoi renvoie le méchant Big Bill de Bison mystique: une instance centrée sur le maintien de l'ordre linguistique comme de l'économie du pouvoir. De ce fait, l'assistant exécute également la règle de la ségrégation du français et de l'anglais dans le spectacle du Projet Rideau Project. Les anecdotes historiques des anglophones réussissent à s'implanter dans l'espace ottavien, que le référent de cet espace soit francophone ou anglophone. Dans The Rhyme, par exemple, la pendaison d'Eugène Larmont a eu lieu à quelques pas de la scène où elle est jouée, les personnages font allusion à la rue Bank et au village avoisinant (et francophone) d'Embrun (Brault 2009: 11, 7; voir note 13). Or, cette implantation réussie ne met que davantage en relief la marginalité, l'atemporalité et l'atopicité des histoires francophones, assurant leur relégation aux marges et aux non-lieux de la ville. La présence franco-ontarienne, contrairement à la présence québécoise, ne renvoie pas le théâtre canadien-anglais «à sa propre problématique d'une identité nationale plus ou moins affirmée» (Harel 1989: 103). Au contraire, le côtoiement du théâtre canadien-anglais et du théâtre franco-ontarien fait réfléchir à la fragilité constitutive de ce dernier dans l'espace et dans le temps.

Le maintien de l'ordre linguistique est aussi mis en évidence dans Peace, Land and Bread. Le français y est non seulement censuré, évacué, mais l'hétérolinguisme s'affiche en son absence, par la présence de la langue russe. Cette langue est parlée autant que le français dans The Rhyme, mais elle est aussi évoquée par un accent russe en anglais et par l'utilisation d'un anglais non accentué dans le dialogue du couple d'origine russe. L'extrait suivant, signalant l'arrivée de l'épouse Svetlana, témoigne de ces stratégies d'écriture:

(8) Igor: [Svetlana, what are you doing? I said to wait outside.] Svetlana: [I'm not just going to sit there.] 
Igor: [Well, this isn't helping me.]

Svetlana: (looking at RAY.) [Is he the one?]

Ray: I'm sorry, I don't... I gather you're with him?

Svetlana opens her mouth to speak.

Igor: [Don't speak.] She is my wife.

$\left(\mathrm{Ng} 2009: 4^{14}\right)$

Dans la pièce, les répliques entre crochets, comme dans l'exemple ci-dessus, sont jouées en anglais sans accent pour évoquer le russe. Celles de Ray, non balisées, sont prononcées en anglais pour référer à cette même langue. Enfin, les répliques d'Igor, en italique, sont jouées en anglais avec un fort accent russe. De telles stratégies de remplacement et d'évocation sont décrites par Lukas Bleichenbacher dans son étude du plurilinguisme cinématographique. Selon lui (2008: 60), les langues autres que la langue principale du film sont souvent évoquées par les accents et le lexique. Dans Peace, Land and Bread, l'accent et le lexique russes en anglais s'opposent toujours à la représentation plus réaliste de l'anglais du commissionnaire. Lorsque les personnages russes conversent entre eux, leur aisance en anglais contraste tout aussi singulièrement avec l'accent laborieux qu'ils adoptent pour parler au commissionnaire. C'est pour assurer la compréhension des spectateurs, et non pour assurer une continuité du réalisme, que le russe lui-même est remplacé par l'anglais. Langue d'immigration, langue du fait divers de la guerre froide, le russe évoque aussi dans cette pièce le multiculturalisme anglo-canadien, c'est-à-dire un multiculturalisme "traduit», évoqué par l'accent, sous la tutelle de l'anglais. Le programme canadien du «multiculturalisme dans un cadre bilingue» (Haque 2012), tel qu'il a été pensé, conçu et entériné dans la Loi sur les langues officielles, est nié par le spectacle, dans lequel le bilinguisme et le multiculturalisme appartiennent aux projets divergents de deux communautés théâtrales distinctes. Le Projet Rideau Project est aussi, à la fin, le spectacle de l'incompatibilité et de l'insolvabilité de ces deux "projets/projects».

\section{Le Projet Rideau Project: hétérolinguisme théâtral et traduction}

Que, des six pièces du spectacle déambulatoire, les deux qui ont fait l'objet d'une traduction soient celles d'ouverture et de clôture, n'est pas sans incidence. Ces deux pièces desservent les festivals dans lesquels s'inscrit le spectacle au moment des représentations, de sorte que les deux tiers des pièces étaient en anglais au Magnetic North Theatre Festival et en français à Zones Théâtrales. Mais ces pièces, Tourist Things et Rebut, sont aussi celles qui comportent un hétérolinguisme minimal. De fait, l'hétérolinguisme pose problème à la traduction et à sa conception habituelle comme transfert entre une langue de départ et une langue d'arrivée (Derrida 1985: 213). De manière plus pratique, les traducteurs butent contre les formes de l'hétérolinguisme, les fonctions qu'il prend et les enjeux qu'il décèle, tant et si bien que de plus en plus de traductologues se penchent sur ces questions (Meylaerts 2006; Suchet 2009; Grutman 2012). Myriam Suchet (2009) propose même un continuum des pratiques de traduction pour les littératures hétérolingues, allant de l'effacement de l'hétérolinguisme à son maintien partiel et à sa transposition. Or si la réflexion sur la traduction de textes hétérolingues a soulevé une attention particulière au cours des dernières années, sa pratique n'en demeure pas moins chargée d'écueils. Qui plus est, la circulation du théâtre hétérolingue exige souvent des stratégies de mise en 
scène et des paratextes (Carlson 2006: 180-213) pour remplacer les pratiques éditoriales (préface, postface, glossaire, etc.) qui sont habituellement l'apanage des traductions de textes littéraires hétérolingues. Le choix de traduire les pièces moins hétérolingues du Projet Rideau Project était peut-être une manière d'éviter ces écueils. Il se pourrait aussi que ces deux pièces assurent un rembourrage de l'hétérolinguisme des autres pièces du spectacle. C'est par le terme cushioning, ou rembourrage en français, que Chantal Zabus (1991: 7) qualifie la glose europhone apposée à un mot africain, stratégie qui, selon elle, sert à nommer et à identifier l'espace «between mother tongue and other tongue without necessarily bridging it». Les traductions de Tourist Things et de Rebut, comme le rembourrage des langues des festivals dans lesquels s'inscrit le Projet Rideau Project, nomment et identifient l'espace théâtral hétérolingue qu'elles contiennent sans pour autant le compléter ou y construire un pont.

À ce titre, la traduction de Tourist Things, Pour les touristes, prépare et anticipe, comme un rembourrage, l'hétérolinguisme des prochaines pièces. Traduite par Paul Lefebvre, traducteur, metteur en scène et conseiller dramaturgique, mais aussi, à l'époque, le directeur artistique de la biennale Zones Théâtrales, la pièce Pour les touristes retient, dans un français populaire canadien, des expressions anglaises du texte de départ. C'est le cas, par exemple, lorsque l'homme interpelle la femme dans l'une de ses premières répliques:

(9) I was - I am - supposed to meet someone here. Lisa. A first date. A blind date. You're sure you're not her?

(Gauthier 2009a: 2; voir note 7)

J'étais - je suis - supposé rencontrer quelqu'un ici. Lisa. Un premier rendez-vous. Un blind date. Vous êtes certaine vous êtes pas elle?

(Gauthier 2009b : 3; traduit par Paul Lefebvre ${ }^{15}$ )

Le blind date est repris du texte de départ, mais il ne détonne pas dans le français populaire de l'homme. Plutôt, l'emprunt est intégré dans la langue d'arrivée, n'engendrant pas d'effet de dépaysement ou d'exotisme. De même, une des répliques récurrentes dans le texte anglais, celle de l'homme qui souhaite prendre une autre photo, est empruntée par la traduction française:

(10) For luck. [...] In case this one didn't turn out

(Gauthier 2009a: 3; voir note 7)

Pour la luck. [...] Au cas celle-là est ratée

(Gauthier 2009b : 5-6; traduction de Paul Lefebvre; voir note 15)

Même les prénoms de l'homme et de la femme (et de son alter ego) témoignent d'une polyvalence linguistique. Ils sont d'abord adaptés par la traduction: de Jess, Amanda et Jennifer (Jen), les noms deviennent Jeff, Anne-Marie et Geneviève (Gen) dans la version française, hormis la traduction, cependant, la diversité linguistique marque déjà le trio de prénoms: Jeff et Jess étant dans les deux cas des prénoms anglais; Gen/Jen faisant le pont entre l'anglais et le français; Amanda et Anne-Marie distinguant ces deux versions. Le français du texte d'arrivée n'est pas celui du texte de départ, où l'homme imitait un accent parisien en anglais dans une conversation au sujet de la tour Eiffel. Plutôt qu'un français parisien, le français du texte d'arrivée 
ressemble davantage à la langue oralisée de l'un des dramaturges franco-ontariens les mieux connus, Jean Marc Dalpé. Comme l'explique la traductologue Louise Ladouceur (2007: 103), les dialogues des personnages de Dalpé «contiennent plusieurs interférences de codes qui reflètent l'inévitable infiltration dont le français fait l'objet dans un contexte bilingue où l'anglais domine». La traduction vers l'anglais des pièces de Dalpé, souvent effectuée avec la collaboration de l'auteur, ne reproduit pas ces interférences sous peine de "sonner faux ou de faire exotique» (Ladouceur 2007: 106). Elle incorpore plutôt, «sans nuire à l'authenticité des dialogues», des bribes de français immédiatement traduites en anglais (Ladouceur 2007: 108). Paradoxalement, aussi bien Tourist Things que Pour les touristes souscrivent à cette logique d'authenticité rembourrée, la première pièce invitant le français sous la forme d'un accent parisien, la deuxième incorporant des interférences lexicales de l'anglais. En ce sens, et en répondant aux attentes de vraisemblance linguistique de son public cible, la traduction de Paul Lefebvre s'intègre assez aisément à la dramaturgie francoontarienne. En outre, le choix des mêmes comédiens pour jouer les personnages de la pièce de départ comme de celle d'arrivée s'avère révélateur du contexte bilingue auquel fait allusion Ladouceur et auquel répondent la dramaturgie de Jean Marc Dalpé... et sa traduction.

«Rembourrant» l'autre extrémité du spectacle et présentée lors du Magnetic North Theatre Festival, la traduction de Rebut sous le titre de Trash est réalisée par Paula Danckert, une dramaturge associée au Théâtre anglais du Centre national des arts d'Ottawa. Incidemment, puisque le Magnetic North Theatre Festival se déroulait en juin 2009 et Zones Théâtrales en septembre de la même année, cette traduction vers l'anglais a été produite avant la pièce qui en était à la source. À cet effet, Rebut contient d'importants changements effectués après les représentations de Trash. Ces modifications affectent surtout le dénouement de la pièce: dans Trash, la dame décapite sa poupée puis s'échappe des confins de la benne, poignard à la main, prête à l'attaque; dans Rebut, la dame enferme les spectateurs dans l'espace clôturé puis agresse la femme charitable devant leurs yeux. Outre cette modification au dénouement, les comédiennes qui jouaient la dame (Tania Lévy) et la femme (Maxine Turcotte) dans Trash échangent leurs rôles pour Rebut. Dans le chassé-croisé de la pièce d'arrivée et de la pièce de départ, ainsi que de leurs représentations respectives, les lignes se brouillent entre l'original et la traduction. À ces changements majeurs s'ajoutent quelques différences mineures apportées par la traductrice. Loin des enjeux de la traduction de l'hétérolinguisme, pour cette pièce de théâtre à la langue assez détachée de la représentation populaire, Paula Danckert doit plutôt négocier la traduction de l'humour de la mendiante, particulièrement prégnant lorsqu'elle tente de divertir un public pour quelques sous.

(11) (Elle s'adresse à quelqu'un dans la foule en tendant son gobelet:)

Excusez-moi, vous connaissez l'histoire de la fille qui essayait de mendier près de la rivière?

(pause)

Ses efforts sont tombés à l'eau!

(À quelqu'un d'autre:)

Celle du guenilleux, vous l'avez entendue? Elle est tordante!

(Avec de moins en moins de confiance:)

Mais pas aussi crevante que celle du sans-abri dans le banc de neige, cependant...

(Migneron 2009a: 3; voir note 8) 
(She addresses someone in the crowd holding out her cup:)

Did you hear about the homeless people lining up at the mission? The police said it was a riot. Did you hear about the panhandler who left Ottawa? He said he was looking for change. Did you hear about the homeless woman who built her shelter with plastic bags instead of cardboard? She was feeling boxed in.

(Migneron 2009b: 3 ; traduit par Paula Danckert ${ }^{16}$ )

Devant un humour qui dépend de constructions linguistiques spécifiques (Antonopoulou et Nikiforidou 2009), la traductrice adapte le spectacle d'humoriste de la dame en ajoutant des références explicites à la ville et en reconstruisant des associations linguistiques afin de déclencher le rire de son public anglo-ottavien. Son approche est donc tout aussi orientée vers la cible que celle de Paul Lefebvre, quoique la cible change dans les deux cas. Confirmant cette approche axée vers la cible théâtrale anglo-ottavienne, Paula Danckert clarifie à quelques reprises des noms et des pronoms ambigus.

(12) Pendant longtemps, la jeune fille, devenue une noble dame, endura le despotisme du dragon - jusqu'à ce que sa bonne fée marraine lui apparaisse, déguisée en camion porteur de déchets bien plus ignobles que celui dont elle s'apprêtait à libérer le monde. La fée marraine profita de la vanité du dragon, qui poussait ce dernier à croire naturel que la circulation s'arrête, ébahie, devant lui. Elle fonça sur lui et d'un seul coup, le dragon disparut, et avec lui les liens qui garrottaient la dame et son enfant.

(Migneron 2009a: 4; voir note 8; notre italique)

The young girl, having become a Gentle Lady, endured the tyranny of the Dragon for a long time - until her fairy God Mother appeared to her disguised as a garbage truck carrying trash more vile than the Dragon Maestro himself. The fairy Godmother took advantage of the Dragon Maestro's vanity for he believed it was natural that the traffic would stop, astonished, in front of him. The garbage truck rammed herself at the dragon and he instantly disappeared, and with him the bonds that tied the Gentle Lady and her child to him.

(Migneron 2009b: 6; traduit par Paula Danckert; voir note 16 ; notre italique)

Le pronom ambigu «elle» est explicité par «The garbage truck», une modification qui répond à la deuxième tendance déformante du traductologue Antoine Berman (1999), la clarification (ou explicitation). Sont aussi explicitées les références à la dame, devenue de manière cohérente dans la version anglaise de la pièce «The Gentle Lady», et celles au dragon, auxquelles le référent est apposé dans «Dragon Maestro». Comme une autre forme de rembourrage nommant et identifiant les espaces trop métaphoriques du texte francophone, ces explicitations des rapports entre l'histoire racontée par la mendiante et son autobiographie contribuent à faciliter la compréhension du public anglophone.

\section{Le Projet Rideau Project: mises en scène et cultures théâtrales}

C’est peut-être justement ce dernier enjeu, celui de la métaphore et des configurations du réel, qui frappe au cœur des questions de la traduction théâtrale qui animent le Projet Rideau Project. On s'en souvient, le directeur artistique du projet, Joël Beddows, 
exprimait qu'il espérait "raconter des histoires [...] sur la "façon" dont les deux groupes culturels et les artistes choisissent aussi de raconter ces histoires ${ }^{17}{ }$. Cette «façon» de raconter des histoires dans les communautés théâtrales francophone et anglophone d'Ottawa est elle-même culturelle. La traductrice de théâtre Linda Gaboriau décrit ces cultures distinctes du théâtre anglo-canadien et québécois de manière éloquente:

When translating Quebec drama for English-speaking audiences I have to bear in mind that I'll be reaching the audience through actors and directors trained and working in English Canada. Actors in English Canada receive training which is distinctly North American and which is often, in terms of contemporary theatre, more psychological than it is in Quebec. They are not as comfortable, for instance, with flights of language, with poetry, or lyrical, rhetorical material. These are precisely the elements which have been the most characteristic of Quebec theatre in the last two decades [1975-1995]. Quebec playwrights have no scrupules [sic] about writing rhetorical sorties, and sometimes English-Canadian actors who have been trained for a different performance style find it quite difficult to handle these texts. (Gaboriau 1995: 84)

À ces distinctions générales entre une culture de théâtre psychologico-réaliste canadienne-anglaise et une culture de théâtre francophone axée sur la langue et la poétique, Louis Patrick Leroux (2012) ajoute que, dans le théâtre québécois contemporain, la recherche du réel trouve actuellement un lieu fécond dans le corps du comédien plutôt que dans la représentation linguistique. Contrairement aux commentaires de Beddows pour qui les «différences» de style et des rapports avec la pratique théâtrale "représentent en soi un creuset riche à explorer" (voir note 17), Gaboriau (1995: 84) fait valoir que les résultats de la traduction théâtrale sont souvent «quite awful, something like the effects of a dubbed movie». C'est contre ces échecs répétés de la traduction dans les croisements de culture théâtrale au Canada, et dans l'optique d'une rencontre renouvelée et fructueuse, que Beddows attribue les textes francophones aux metteurs en scène anglophones et vice versa: Jean Stéphane Roy pour Tourist Things/Pour les touristes, Elif Işıközlü pour Cercles polaires, Benoit Roy pour Peace, Land and Bread, Kevin Orr pour Bison mystique, Natalie Joy Quesnel pour The Rhyme of Nicholas Street Goal et Joël Beddows lui-même pour Rebut/Trash.

Pour les metteurs en scène francophones, c'est-à-dire Jean Stéphane Roy, Benoit Roy et Natalie Joy Quesnel, le défi est peut-être d'incorporer au texte une distance ou une métaphore scénique. Jean Stéphane Roy transforme ainsi le texte de Tourist Things/Pour les touristes en comédie musicale. Ses comédiens se chantent alors la pomme sur l'air de Downtown, chanson pop composée par Tony Hatch à la suite d'une première visite de New York et popularisée par Petula Clark: «When you're alone and life is making you lonely / You can always go, downtown / [...] / So listen to the music of the traffic in the city ${ }^{18} »$. En plus de faire en sorte que les comédiens accaparent l'espace urbain par la danse, cette chanson réitère les enjeux principaux du spectacle comme de la pièce. Ainsi, elle évoque l'urbanité explorée par le Projet Rideau Project et le désir de la femme de rencontrer un étranger dans un espace public. De plus, la chanson Downtown a servi à la campagne new-yorkaise visant à attirer les touristes après les attentats du 11 septembre 2001, ce qui ajoute un clin d'œil ironique au refus de l'homme de parler aux touristes.

De cette mise en scène, le critique de théâtre du Ottawa Citizen, Patrick Langston, dit cependant qu'elle «feels contrived, as though playwright and actors John Doucet, 
Julian Doucet and Andrée Rainville need to workshop it a time or two ${ }^{19}$ ». Pour sa part, la critique francophone Dominique Lafon approuve l'apport musical de la mise en scène: "Malgré le bruit du trafic qui couvrait parfois leurs voix, [les comédiens] surent garder le rythme d'un duo romantique monté comme un clin d'œil à la comédie musicale ${ }^{20}$.» Elle fait aussi une critique élogieuse de The Rhyme of Nicholas Street Goal, dont elle dit que «le huis clos oppressant des lieux offrait à la mise en scène la possibilité d'effets sonores (coups frappés contre une porte de métal) et visuels (noir complet pendant les premières minutes de la représentation) qui tout en étant convenus n'en demeuraient pas moins efficaces». Langston, lui, conclut qu'il s'agit de "one of the finest pieces in the project» et d'un "model of site-specific theatre » aux côtés de Peace, Land and Bread. Lafon est loin d'être en accord au sujet de cette dernière pièce, parlant d'«un cadre hyperréaliste mais bien peu théâtral» et de «la reconstitution sans relief d'un épisode de la guerre froide: on était plutôt dans la vignette naturaliste malgré la générosité de l'interprétation». Des trois metteurs en scène, Benoit Roy prend le moins de distance métaphorique du réalisme du texte, pour le plaisir de la critique anglophone mais au chagrin de la critique francophone.

Inversement, pour les metteurs en scène anglophones, un des grands défis était de relier à la ville - ou à un autre aspect du réel - les textes déracinés des auteurs francophones. Pour Elif Işıközlü, l'ancrage dans l'espace de Cercles polaires autour des statues de l'ours polaire et de la fille au cerceau, déjà évident dans la langue des personnages de Ouellette, se prolonge dans le mouvement des comédiens. Benjamin Gaillard adopte ainsi la posture déséquilibrée de l'ours polaire pour se déplacer; Annie Lefebvre fait une danse au cerceau; la rencontre des deux mouvements forme une pièce dont les éléments clownesques, selon Langston, «underscore its theme of human alienation». Lafon, cependant, considère que le prolongement gestuel des sculptures présentes sur les lieux ne suffisait pas: "paradoxalement, ni l'ingéniosité de la mise en scène ni l'énergie de l'interprétation ne parvenaient à faire prendre forme à un texte dans lequel l'auteur faisait surtout montre de sa virtuosité verbale». Bison mystique souffre selon elle d'une faiblesse dramaturgique semblable, que le metteur en scène, «jouant le tout pour le tout et allant chercher chez Beckett au moins un cadre de référence, chercha à faire oublier». Incidemment, Langston relève les allusions scéniques beckettiennes («one character suffers from sore feet, a wilted carrot appears») et signale la réflexion sur la transitivité de l'espace public auxquelles elles ont donné lieu. C'est cependant pour Rebut/Trash et sa mise en scène par Joël Beddows que les deux critiques gardent leurs commentaires les plus virulents. Lafon explique que cette dernière pièce du spectacle, qu'elle a vue en français,

faisait figure de corps étranger en raison d'un parti pris scénographique totalement en rupture avec l'esthétique de l'ensemble du projet. Loin d'exploiter le lieu pour ce qu'il était, un renfoncement dans l'allée entre la Cour des Arts et l'ancienne prison, la scénographie installait un décor symbolique qui déconstruisait la pertinence de la mise en place et l'excès métaphorique d'un texte qu'un dénouement plaqué in extremis ne sauvait pas de l'artifice malgré les efforts déployés par la comédienne pour établir le contact avec le public. (Lafon 2009-2010; voir note 20)

$\mathrm{Ou}$, comme le résume adroitement Langston, «more narrative, less metaphor». C’est la rupture avec le réel - pour Lafon, celui de l'espace; pour Langston, celui de la narration - au profit d'un surplus de métaphores qui semble faire échouer la mise en scène de cette pièce. Or, comme le souligne Beddows dans son entretien avec 
Alvina Ruprecht, il y avait une réelle matérialité au spectacle: «le lieu n’était pas seulement sordide et lugubre, mais très sale. Cela sentait l'urine partout mais j'ai insisté pour qu'on ne nettoie pas l'endroit parce que la saleté figurait dans la symbolisation du personnage principal» (Ruprecht 2010: 297; voir aussi Nutting 2012: 24). Cette composante de la mise en scène du réel ne semble cependant pas avoir figuré dans l'expérience des spectateurs et critiques. Et puisque Trash, ou Rebut selon les représentations, clôturait le spectacle du Projet Rideau Project, il y a lieu de se questionner sur le succès des rencontres théâtrales générées par celui-ci.

À ce sujet, le Projet Rideau Project n'est peut-être que l'un des premiers jalons de l'histoire des rencontres entre communautés théâtrales ottaviennes. En août 2010, à la même Cour des arts qui accueillait The Rhyme of Nicholas Street Goal (la pièce qui semble avoir eu le plus grand succès), se jouaient de telles rencontres. D'une part, la pièce bilingue Inseparable de Louis Lemire, mise en scène par Matthew Romantini, était à l'affiche avec un comédien francophone et un autre anglophone. D'autre part, Beddows présentait un nouveau projet, la pièce Swimming in the Shallows, dans lequel il invitait des comédiens francophones à jouer en anglais. Et le metteur en scène d'ajouter: "Il y a 12 ans, quand j'ai commencé à Ottawa, je n'aurais jamais cru que de telles rencontres entre les deux milieux de théâtre pourraient avoir lieu. C'est très beau, ce qui nous arrive depuis le Projet Rideau de l'an dernier ${ }^{21}$.»

\section{Hétérolinguisme, co(-)linguisme ${ }^{22}$ et bilinguisme officiel}

Au terme de ces déambulations dramaturgiques et traductologiques et d'un parcours des mises en scène du Projet Rideau Project, quelques mises au point terminologiques et conceptuelles s'imposent. Jusqu'à maintenant, le terme «hétérolinguisme» m’a été utile pour décrire l'investissement dramaturgique de l'hétérogénéité des langues et des sociolectes dans le Projet Rideau Project. Outil d'analyse littéraire plutôt que sociologique, l'hétérolinguisme «contredit nos habitudes de lecture (et de traduction), lesquelles font l'impasse sur les rapports complexes que les textes littéraires entretiennent avec le monde extratextuel» (Grutman 2012: 52). Or, mon usage du terme «hétérolingue», il faut l'admettre, contredit l'appellation "co-lingue» attribuée au spectacle. Dans les milieux théâtraux canadiens et québécois, cette désignation a surtout servi à décrire le fonctionnement de l'École nationale de théâtre du Canada/ National Theatre School of Canada, une institution qui offre les mêmes formations, en parallèle, en français et en anglais, sans que les étudiants des deux programmes n'aient à se côtoyer. Ce co-linguisme n'est pas sans rappeler la Loi sur les langues officielles, dont l'objectif n'est pas le bilinguisme généralisé de la population mais l'offre de services dans la langue officielle de l'individu, permettant à ce dernier de demeurer unilingue si tel est son désir. Ainsi, bien que l'un des objectifs originaux de l'école ait été le partage artistique translinguistique, il reste que son succès demeure partiel «for obvious political reasons» (Salter 1992: 8), le programme de scénographie étant le seul à cultiver le genre d'échange "co-lingue» visé dans la formation et dans la création.

Pour Beddows, à qui j'ai demandé des clarifications lors d'un entretien ${ }^{23}$, le théâtre co-lingue, comme l'hétérolinguisme, cherche à différencier le milieu (bilingue) de l'œuvre artistique; il désigne le travail en voies parallèles de différentes langues, en ouvrant ces voies à des zones de contact là où l'esthétique le permet, mais sans 
que ce soit gratuit. Le co-linguisme, ajoute-t-il, est aussi la rencontre de deux cultures théâtrales. À titre d'exemple, il se réfère à la mise en scène de Tourist Things par Jean Stéphane Roy qui, selon lui, pose sur l'araignée un regard extérieur alors que le texte de Gauthier suggérait une vue sur la ville à partir de l'intérieur. Beddows relève chez le metteur en scène une allégorie de la perspective du Québec qui croit qu'on le regarde en tout temps. Ce dernier commentaire sur la portée allégorique de la pièce (et par extension, du spectacle) laisse comprendre la complexité de ses rapports avec le monde extratextuel de la ville d'Ottawa, avec ses idéologies et avec les sites qui l'exposent au «réel». L'accommodement linguistique du spectacle selon la langue du festival dans lequel il s'inscrivait contribue également à brouiller ces rapports et à rapprocher le co-linguisme théâtral de Beddows du «colinguisme» défini par Renée Balibar (1993: 7) comme «l'association, par l'enseignement et la politique, de certaines langues écrites faisant communiquer des partenaires légitimes ». Dans le Projet Rideau Project, la traduction agit aussi comme mode associatif faisant communiquer des partenaires légitimes, c'est-à-dire les festivals de théâtre construits selon un modèle unilingue.

Mais qu'il soit présenté lors d'un festival francophone ou anglophone, le colinguisme du Projet Rideau Project se distingue du bilinguisme officiel canadien en ce que la traduction ne permet pas de garantir l'unilinguisme des spectateurs. La popularité du spectacle auprès des spectateurs de la communauté francophone (plus bilingue que la communauté anglophone) le signale d'ailleurs aussi. Beddows confirmait lors de l'entretien que les commentaires élogieux de la critique francophone (précédant Lafon) ont encouragé les spectateurs francophones ou bilingues à assister aux représentations visant un public anglophone. En outre, pour les spectateurs unilingues, les réseaux linguistiques établis en parallèle laissent peu de place à la traduction si ce n'est que par l'entremise des guides qui accompagnent les groupes d'un environnement scénique à l'autre. Comme les fonctionnaires fédéraux appelés à servir les citoyens dans la langue de leur choix, ces guides, qui rappellent le théâtre patrimonial et touristique, assurent un minimum de compréhension pour les spectateurs qui n'auraient pas accès aux deux langues du spectacle. Cet élément humain, dialogique et spontané, s'apparente à un autre sens du «colinguisme», plus récent et lié au monde universitaire plutôt qu'à celui de la pratique des lettres et du théâtre. La critique et traductologue Catherine Leclerc (2010) se sert ainsi du terme "colinguisme» pour évoquer une exigence de parité et de réciprocité dans les zones de contact des langues littéraires. Dans le Projet Rideau Project, ce sont les guides qui assurent cette réciprocité auprès des spectateurs unilingues éventuels, prenant contact avec eux et avec leurs exigences linguistiques. Ils pourraient ainsi établir un nouveau pacte de traduction. Cette composante de la réception répondrait aux objectifs de co-linguisme de la production, faisant résonner l'échange des spectateurs avec celle des artistes et créateurs, alors même que les poétiques hétérolingues des pièces anglophones et francophones s'entrechoquent et se répondent, que leurs poétiques de traduction soulèvent un manque à combler en termes de réciprocité et de parité.

\section{REMERCIEMENTS}

Je tiens à remercier Joël Beddows, Maurice Demers et Sylvain Sabatié pour les matériaux (dossier de presse, textes dramatiques, captations vidéo) du Projet Rideau Project qu'ils ont aimablement mis à ma disposition à la suite de mon entretien avec Joël Beddows le 9 août 2012. 


\section{NOTES}

1. Il ne s'agit pas tant de deux groupes ethniques monolithiques mais plutôt de deux milieux professionnels contigus alimentés par des artistes d'origines diverses qui s'y associent sur la base de la langue de travail. Sans les effacer, on ne s'arrêtera pas ici aux artistes qui s'excluent (ou qui sont exclus) de ces deux milieux majeurs du théâtre professionnel à Ottawa.

2. La graphie du titre alterne entre Projet Rideau/Rideau Project et Projet Rideau Project. Par souci de cohérence, je parlerai, comme le fait le directeur artistique du Théâtre la Catapulte de l'époque, du Projet Rideau Project. On pourrait tout aussi bien lire dans le maintien de la barre oblique et de l'effet de traduction du titre un clin d'œil intéressant à une œuvre phare de la littérature francoontarienne, L'homme invisible/The Invisible Man de Patrice Desbiens, publiée pour la première fois en 1981.

3. Dossier de presse (2009): Projet Rideau/Rideau Project. Ottawa: Théâtre la Catapulte.

4. Beddows, Joël (2009) : Mot de Joël Beddows, directeur artistique. Dossier de presse: Projet Rideau/ Rideau Project. Ottawa: Théâtre la Catapulte.

5. Commission de la capitale nationale. À propos de la CCN. Mandat et organisation. Consulté le 24 juin 2013, www.capitaleducanada.gc.ca/a-propos-de-la-ccn/mandat-organisation

6. D'après le titre de l'ouvrage de Rainier Grutman (1997) sur l'hétérolinguisme au XIXe siècle québécois (voir section Références).

7. Gauthier, Patrick (2009a): Tourist Things. Ottawa: Théâtre la Catapulte.

8. Migneron, Sarah (2009a): Rebut. Ottawa: Théâtre la Catapulte.

9. Ouellette, Michel (2009): Cercles polaires. Ottawa: Théâtre la Catapulte.

10. Moquin, Luc (2009): Bison mystique. Ottawa: Théâtre la Catapulte.

11. Maillet, Antonine (1971): La Sagouine: pièce pour une femme seule. Montréal: Leméac.

12. La chanson des Prairies. 3600 secondes d'extase (4 novembre 2010): réalisé par Isabelle Garneau et Pierre Ouimet. Montréal: Radio-Canada.

13. Brault, Pierre (2009): The Rhyme of Nicholas Street Goal. Ottawa: Théâtre la Catapulte.

14. NG, John (2009): Peace, Land and Bread. Ottawa: Théâtre la Catapulte.

15. Gauthier, Patrick (2009b): Pour les touristes. (Traduit par Paul Lefebvre). Ottawa: Théâtre la Catapulte.

16. Migneron, Sarah (2009b): Trash. (Traduit par Paula Danckert). Ottawa: Théâtre la Catapulte.

17. Beddows, Joël (2009) : Mot de Joël Beddows, directeur artistique. Dossier de presse: Projet Rideau/ Rideau Project. Ottawa: Théâtre la Catapulte.

18. Downtown (1964): Composée par Tony Hatch et interprétée par Petula Clark.

19. Langston, Patrick (6 juin 2009): Theatre Review: The Rideau Project. The Ottawa Citizen. Consulté le 6 juin 2013, www.ottawacitizen.com/entertainement/story.html?id=1670472

20. Lafon, Dominique (Hiver 2009-2010): Le projet Rideau / Rideau Project. Liaison. 146: 24-25.

21. Beddows, Joël, cité dans Valérie Lessard (7 août 2010): Deux solitudes s'affrontent et se fondent sur les planches. Le Droit: A12.

22. Pour écrire «co-linguisme», certains auteurs se servent du trait d'union, d'autres, non. Habituellement, ce sont les chercheurs qui laissent tomber le trait d'union alors que les artistes et administrateurs s'en servent. Il s'agit dans la plupart des cas d'usages (para)synonymiques.

23. Propos recueillis le 9 août 2012 lors d'un entretien avec Joël BeDDows, ancien directeur artistique du Théâtre la Catapulte.

\section{RÉFÉRENCES}

Antonopoulou, Eleni et Kiki Nikiforidou (2009): Deconstructing Verbal Humour with Construction Grammar. In: Geert Brône et Jeroen Vandaele (dir.), Cognitive Poetics: Goals, Gains and Gaps. Berlin; New York: Mouton de Gruyter, 289-314.

Balibar, Renée (1993): Le Colinguisme. Paris: Presses universitaires de France.

BedDows, Joël (2001): Tracer ses frontières: vers un théâtre franco-ontarien de création à Ottawa. In: Hélène Beauchamp et Joël Beddows (dir.), Les Théâtres professionnels du Canada francophone: entre mémoire et rupture. Ottawa: Le Nordir, 49-69.

Berman, Antoine (1999): La Traduction et la lettre: ou l'Auberge du lointain. Paris: Seuil.

BleichenbaCher, Lukas (2008): Multilingualism in the Movies: Hollywood Characters and Their Language Choices. Tübingen: Francke. 
Brun del Re, Ariane (2012): À la croisée de la Côte de Sable et de King Edward: Ottawa, capitale littéraire de l'Ontario français? Francophonies d'Amérique. 34:105-135.

CARlson, Marvin A. (2006): Speaking in Tongues: Language at Play in the Theatre. Ann Arbor: University of Michigan Press.

Derrida, Jacques (1985): Des Tours de Babel. In: Joseph F. Graham (dir.), Difference in Translation. Ithaca: Cornell University Press, 209-248.

Gaboriau, Linda (1995): The Cultures of Theatre. In: Sherry Simon (dir.), Culture in Transit: Translating the Literature of Quebec. Montréal: Véhicule Press, 83-90.

Grutman, Rainier (1997): Des langues qui résonnent: l'hétérolinguisme au XIX ${ }^{e}$ siècle québécois. Saint-Laurent: Fides.

Grutman, Rainier (2012): Traduire l'hétérolinguisme: questions conceptuelles et (con)textuelles. In: Marie-Annick Montout (dir.), Autour d'Olive Senior: hétérolinguisme et traduction. Angers: Presses de l'Université d'Angers, 49-81.

Haque, Eve (2012): Multiculturalism Within a Bilingual Framework: Language, Race, and Belonging in Canada. Toronto; Buffalo: University of Toronto Press.

HAREL, Simon (1989): Le voleur de parcours: identité et cosmopolitisme dans la littérature québécoise contemporaine. L'Univers des discours. Longueuil: Le Préambule.

Нотте, Lucie (2000): Entre l'être et le paraître: l'altérité et l'identité dans deux textes francoontariens, In: Yvan G. Lepage et Robert Major (dir.), Croire à l'écriture. Études en littérature québécoise en hommage à Jean-Louis Major. Ottawa: David. 163-178.

Нотте, Lucie (2002): La littérature franco-ontarienne à la recherche d'une nouvelle voie: Enjeux du particularisme et de l'universalisme. In: Lucie Нотте (dir.), La Littérature francoontarienne: voies nouvelles, nouvelles voix. Sudbury: Prise de parole, 35-47.

Ladouceur, Louise (2007): Parler, écrire et traduire dans la langue de Dalpé. In: François Paré et Stéphanie Nutting (dir.), Jean Marc Dalpé: ouvrier d'un dire. Sudbury: Prise de parole, 97-112.

LeCLERC, Catherine (2010): Des langues en partage? Cohabitation du français et de l'anglais en littérature contemporaine. Montréal: XYZ éditeur.

Leroux, Louis Patrick (2012): From langue to Body: The Quest for the «Real» in Québécois Theatre. In: Roberta BARker et Kim Solga (dir.), New Canadian Realisms. Toronto: Playwrights Canada Press, 106-123.

Meylaerts, Reine (2006): Heterolingualism In/and Translation: How Legitimate Are the Other and His/her Language? An Introduction. Target. 18(1):1-15.

Nutting, Stéphanie (2012) : Joël Beddows, agent double. Francophonies d'Amérique. 33:13-35.

Ruprecht, Alvina (2010): Le théâtre franco-ontarien: Entretien avec Joël Beddows, directeur artistique du Théâtre la Catapulte, Ottawa, Canada. International Journal of Francophone Studies. 13(2):287-314.

Salter, Denis (1992): Body Politics: English-Canadian Acting at National Theatre School. Canadian Theatre Review. 71:4-14.

Suchet, Myriam (2009): Outils pour une traduction postcoloniale: littératures hétérolingues. Paris: Archives contemporaines.

Zabus, Chantal (1991): The African Palimpsest: Indigenization of Language in the West African Europhone Novel. Amsterdam: Rodopi. 